\title{
Non-Invasive Blood Glucose Measurement Technique for Diabetic Patients
}

\author{
S. Priya Dharshini, M. Revathy, A. Soundharya, R. Manjula Devi
}

\begin{abstract}
Diabetes mellitus is one of the most spreading diseases prevalent in the world. The Diabetic patients where in desideratum of monitoring their blood glucose level conventionally for a certain periodic of time. If they were nescient of their checkup it may lead to sundry symptoms like the feel profoundly slothful, tired etc. So they require for a periodic check up without fail. In clinic the prefer syringes for amassing the samples, in some worst cases there will be a possibility of unhydrated syringes. For checking they were in desideratum of a modicum of blood samples. For a precise quantification they will integrate some chemical substance to detect the value. While taking blood samples they feel so uncomfortable, pain and if the syringes where already used are not felicitously sterilized it may lead to sundry disease. The another method for quantifying the sample is a non-invasive. In non-invasive method there will be no desideratum of any syringe. It will be pain less and withal comfortable for the patients. In this paper we are introducing a non-invasive method in lieu of invasive method to be liberate from pain. Our proposed system consists of near infrared transmitter and receiver, a light dependent resistor etc,. The rays are sanctioned to passes through the finger it will quantify the glucose present in our cell and the output is given to the processor. The processed output will determine the quantity of glucose present in the blood. Then determinately the obtained output will be exhibited in the Liquid Crystal Exhibit.
\end{abstract}

Keywords: - NIR Transmitter and Receiver, Arduino Uno, Serial Monitor.

\section{INTRODUCTION}

Diabetes is one of the types of metabolic diseases. For diabetic patients the sugar level in the blood is increased drastically from its normal level. Generally for diabetic patients the sugar level in human body increases due to inadequate segregation of insulin in blood cells or because of infelicitous copying of body cells to the insulin. Diabetes can create most important impediment such as heart attack and optical incapacitation in the human body. So it is serious to monitor the glucose level.The World Health Organization (WHO) estimated that there are 200 million diabetic patients are there in the world wide. Diabetes is a blatant of a body where it is not able to predict the amount of insulin required to maintain ordinary level of blood glucose.

Revised Manuscript Received on February 05, 2020.

* Correspondence Author

S. Priya Dharshini, Undergraduate student in department of Electronics and communication Engineering, National Engineering College, Tamil Nadu, India.

M.Revathy, Student, Department of Electronics and communication engineering at National Engineering College, Tamil Nadu, India.

A. Soundharya, Undergraduate student in Department of Electronics and communication engineering at National Engineering College, Tamil Nadu, India.

R.Manjula Devi, Assistant Professor (SG) in the Department of Electronics and Communication Engineering at National Engineering College, Tamil Nadu, India.

(c) The Authors. Published by Blue Eyes Intelligence Engineering and Sciences Publication (BEIESP). This is an open access article under the CC BY-NC-ND license (http://creativecommons.org/licenses/by-nc-nd/4.0/)
So, it is necessary for the diabetic patients to regulate the blood glucose levels through congruous diet or they should inject the required level of insulin. For the effective treatment of diabetes, patients have to quantify the caliber of blood glucose periodically.

At present, most of the diabetic patients are make use of invasive figure piercing instrument such as glucose meter to acquaintance the concentration of blood glucose.

Development of a non-invasive glucose quantification technique will be boon for a diabetic patient. The most important advantage of non-invasive quantification methods is the mitigation from pain and placate due to no finger puncturing. The monitoring of glucose level using non-invasive method truncates the difficulties involved in glucose quantification and hence minimizes the cost of healthcare.

For the last decade there are several literatures were carried out in the non-invasive blood glucose measurement. The researchers are utilizing sundry optical methods for the non-invasive quantifications which includes near-infrared, photo acoustic spectroscopy, Raman's spectroscopy, polarization technique and light scattering techniques, Trans-illuminated laser beam is utilized to quantify glucose concentration as described by Tang, metabolic heat conformation technique can be additionally utilized for blood glucose quantification. In the Near Infrared (NIR) Spectroscopy, glucose cells will engender the most impuissant NIR absorption signals in the human body. The quantification of the glucose level using NIR spectroscopy facilitates the infiltrate the signal inside human tissue within the range of 1 to $100 \mathrm{~mm}$ depth. Perforation depth decreases as the signal wavelength value increases. Recent literature stated that the non invasive method of glucose measurement incorporated neural network techniques. In this work, the quantification of blood glucose using non invasive method surmounts. The glucose optical measurement techniques may be classified into the following Subclasses such as Near Infrared Spectroscopy (NIR), Infrared Spectroscopy (IR), Raman Spectroscopy, Photo acoustic and opto-acoustic techniques, spatially resolved diffuse reflectance measurements

\section{LITERATURE SURVEY}

A ecological, cheapest test divest for glucose was established by energise by glucose for oxidizing the enzyme and bromocresol purple $\mathrm{pH}$ change and, thus a vicissitude in colour of a sieve paper divest, that was noticed by a standalone electronic meter[1] The non-invasive RF quantification of human blood glucose on the detection of the transfiguration in the permittivity of the blood utilizing a resonator act as a sensor. 
[2 Blood glucose levels such as interchange establishes the acetone by a satisfactory baker for diabetes[3] Quantity using painless near infrared predicated optical technique.The wavelength of LED is about 360-950. The optical visual signals are transmitted throughthe fingertip and reflected signals are detected by phototransistor placed in collocation of the LED [4Near Infrared (NIR) spectroscopy to determine blood glucose levels predicated on transmittance spectroscopy on the auditory perceiver lobe [5] In-vitro glucose quantification archetype is developed utilizing perpetual wave (CW) from NIR LED (940 nm) to check the sensitivity of the system for various glucose absorption [6] . The in-house established meter consist by an visually separated field in the chassis for including the test divest with the small amount of sample to eschew interferences from ambient wind of detection between 32 to $516-\mathrm{mg} / \mathrm{dL}$ glucose concentration in the identification was about 1.0 $\mathrm{mg} / \mathrm{dL}$ /amount of a constraint of detection (LOD) of 32 $\mathrm{mg} / \mathrm{dL}$ with the replication time about $15 \mathrm{~s}$.A contrivance along with divests were validated using a clinical patternfor correlating with the salivary blood glucose level with that of blood glucose level with the help of a trade of glucometer. A known quantity of better comparing is found for diabetic individuals (0.80), whereas it was less consequential for non-diabetic individuals. The contrivance agreed should be medically acceptable. A simple and low-cost setup should be identically suitable used for huge separate of diabetics, to one side from being utilized as a self-monitoring contrivance in a non-invasive and painless manner[7] The non-invasive quantification of body fluid like blood glucose isone of the prevalent investigation issue in which Radio frequency and microwave detecting of blood glucose was the most promising methods in this field. Since there will be a lot of existing quantification sites in human body, fingertips appear to be a good cull because of a substantial quantity of new body fluid source and similarity in terms of a biotic sheets present. The non-invasive RF quantification of blood glucose relies on the detection of the transmutation in the permittivity of the blood utilizing a resonator act as a sensor. Though, the transmutation of the permittivity of the blood glucose because of the difference in the glucose contented has a circumscribed with the range of resulting in a minutely minuscular shift occurs in the sensor's frequency duplication. A little erraticism between quantifications may obstruct the quantification outcomes. The variations are mostly arise due to the variation of thickness in the biotic layers and variation of dactylograms which will be uniquely for every human. Consequently, the variable properties of genetic layers and dactylograms are with the fingertips was studied in elaborate and that are reported [8 TheNon-invasive diabetic prognosticationhave been in advance importance for the past decennium. For most human the vaccine are estimated, for the humanoid breathing develops as a paramount selection for an acetone level for the breathing showing a good correlation to human blood glucose levels. Due to Suchrelationship acetone was acceptable as a biomarker for diabetic patients. The one of most prevalent statistics study policies to investigate the biomarkers in breath for sickness exposure use feature extraction and also relegation processes. Yet, difficulties such as computational cost and also the lack of optimum features cull on tender to genuine-time indicators abbreviate the efficiency of such analysis. The newspaper discovers the utilization of a unidimensional (1-D) changed(CNN) system that amalgamates feature abstraction and relegation systems. The method projected in the paper is found to be meaningfully abbreviate as ainhibitions associated with utilizing these techniques individually and thereby amending the classifier's concert further. The paper advises to put on a changed 1-D CNN on authentic-time the breath signals resulted from an array of the gas sensors. The experimental and the presentation of the scheme is passed out and appraised [9] Diabetes is kenned as one of the spreading diseases in the world that arises not only among adults, elderly, infants and children. Blood glucose quantifications are vital for diabetes patients for determining their insulin dosage taken and perpetual intensive care. The value of glucoseis always withthe mundanevariety. The generally used procedures to quantify glucose results in the blood are invasive which are high in precision but it leads to painful and has higher risk of infections.An substitute method for an non-invasive glucose techniques were announced to progress pain free glucose quantifying methods. A transportable non-invasiveblood glucose monitoring contrivance is developed utilizing near infrared sensors. Several in vitro and in vivo experiments proved the utilizations of the contrivance. The results of the experiments prove that the Non-invasive is better than invasive method [10 Diabetesis one of the major contributors of non-contagious diseases. Prevailing method for tenaciousness of blood glucose concentration is utilizing the self-monitoring glucose equipment. This process includes pricking the blood from the finger and extracting the blood from the forearm and by using the chemical analysis with the avail of one-use test divests. The ache, throe and arduousness caused by this technique have lead to the development of a non-invasive method of quantification predicated on the survey conducted. These are the method makes utilization of a NIR sensor for transmitting and receiving of rays from the fingertip. The Near-infrared (NIR) is sent through the fingertip, afore and after occluding the blood flow. By analyzing both the cases the datas are send to the wirelessly connected PC.[11]

\section{III.BLOCK DIAGRAM}

The proposed system has various modules such as supply, NIR transmitter, finger, IR receiver, Serial monitor. The block diagram of the proposed work is shown in figure.1

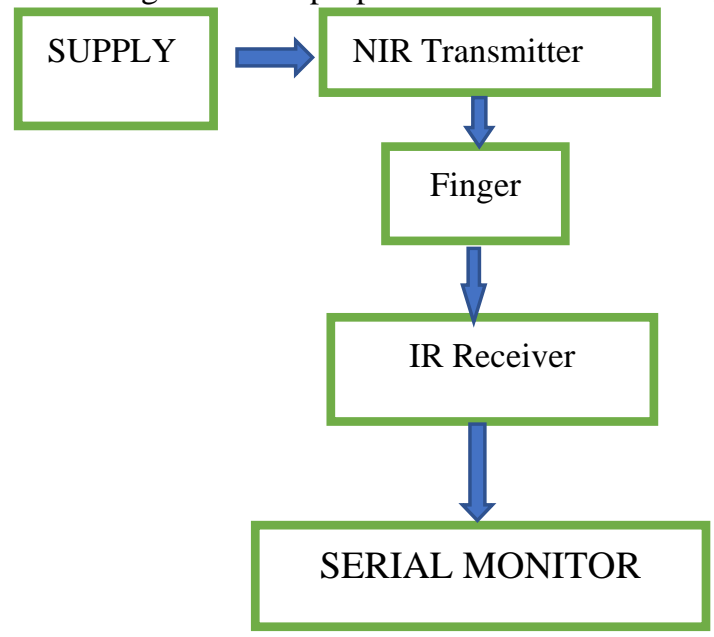

Fig 1 The block diagram of proposed work 


\section{NIR SPECTROSCOPY}

NIR spectroscopy is predicated on amassing reflectance or absorption spectra of the tissue with a spectrometer. When NIR light elucidates into the skin, a scintilla is engrossed and sprinkled as a result of its interaction the chemical mechanism in the tissue illuminate.

The amount of light that was not absorbed will be reflected out of the tissue or transmitted through it. NIR based transmission spectroscopy was used to quantify blood glucose concentrations in diabetic subjects. The glucose penetration of NIR spectroscopy is as shown in figure 2.

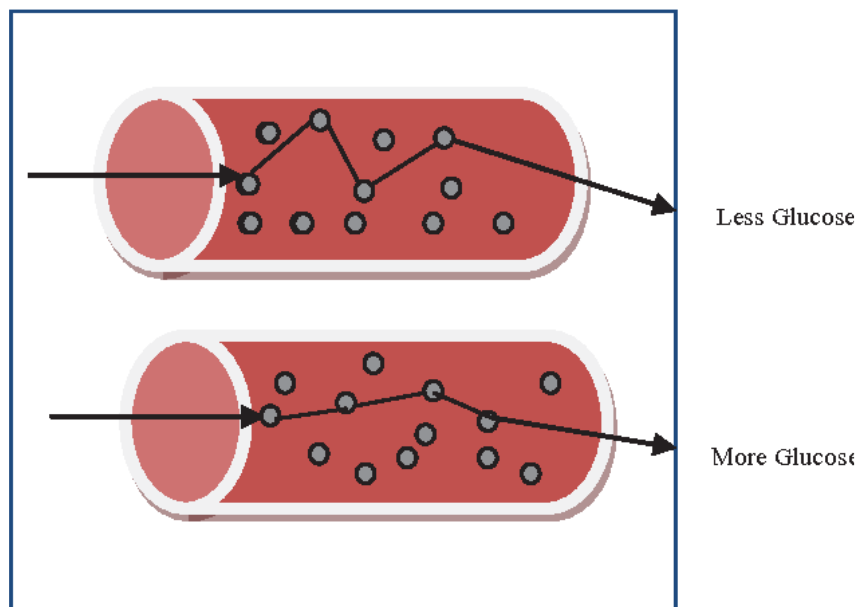

Fig 2 NIR spectroscopy for the glucose penetration

\section{IV.MATERIALS}

Near infrared transmitter is for sensing the glucose present in the blood, The IR transmitter and receiver is utilized for quantifying the glucose level. Arduino Uno is for dumping the code. Output is exhibited in the serial monitor.

\subsection{NIR TRANSMITTER}

The near-infrared ranges from 650 to $1350 \mathrm{~nm}$ where light is perforating into the tissue. Medical imaging techniques make utilization of the NIR window to detect deep structures.

\subsection{IR RECEIVER}

These types of sensors are of reflective property. The emitter emits beam which is reflected by the object.

\subsection{ARDUINO}

The ARDUINO is an microcontroller kit utilized for designing and to build the authentic time environment. It has 14 digital input/output pins, 6 analog inputs, a $16 \mathrm{MHz}$ ceramic resonator, a USB connection, a potency jack, an ICSP header, and a reset button.

4.4.IC 741 Operational Amplifier: The IC 741 operational amplifier looks akin to a minuscule chip. The op-amp IC 741 consists of eight pins. The most paramount pins is pin 2 and 3 denote inverting \& non-inverting terminals and pin6 denotes output voltage. The triangular form in the IC betokens an op-amp integrated circuit

\section{V.PROPOSED SYSTEM}

Beer-lamberts law states the relationship between absorbance and concentration of absorbing spices. When IR light is incident on material part of it is absorbed by the molecules. The absorption of IR light is different for different wavelength. It exploits Near Infrared transceiver to conclude the blood glucose levels predicated on the Potation-Lambert's Law. With the benefit of employing sundry parameters, such as tissue thickness, blood oxygen saturation, and a linear regression analysis predicated calibration system, a precise and genuine-time architecture is proposed. Near Infrared Transceiver is utilized across the finger to quantify glucose. Near Infrared Transceiver involves a light sources and a light detector situated on either side of the finger. The quantity of near infrared light passing through the finger depends on the magnitude of blood glucose in that region. Near-Infrared(NIR) light is applied on the either side of the finger, some light will be absorbed and some will be reflected predicated on the glucose concentration in that region. The reflected signal is then processed and analyzed. In addition to the blood glucose measurement, the transmittance of NIR light depends on the amount of blood that travel in the path of light. The same glucose levels, a generously voluminous magnitude of blood result in lower transmittance, whereas less blood flow results in an astronomically enormous transmittance. During the time of quantification, The value of glucose level needs to be scaled. Initially, the NIR transceiver is placed on either side of the finger, then the attenuated light is received by the photodiode placed at other side of finger. This received signal is amplified and filtered for noise by first and second order filtering. It is processed to determine the characteristics of signal. Determinately the glucose level is resolute and it is exhibited on LCD.The proposed model of our work is as shown in figure 3.

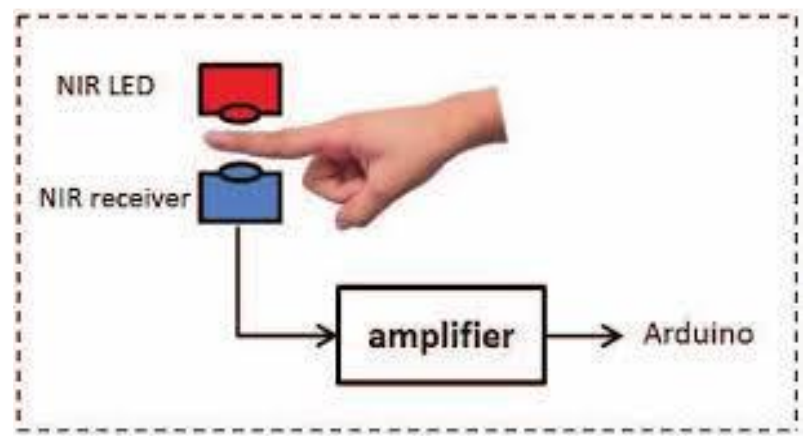

Fig 3. The Proposed model of our work.

Fiber optical bundles connect light source (tungsten halogen lamp) and spectrometer with the fiber optical quantifying head. The fibers lighten the finger tip then the circularly arrangements are around the central part of the bundle which will connect with the spectrometer. They calculate the calibration coefficients and by recording the spectra from sundry diabetic patients with varying BG concentration levels. The BG level is resolute simultaneously by a conventional glucose analyzer utilizing blood plasma. A typical blood glucose profile is shown in Fig 4. Diffuse reflectance studies of the inner lip additionally have shown good correlation with blood glucose and designated a time lag of 10 minutes between blood glucose and the quantification signal. 


\section{Non-Invasive Blood Glucose Measurement Technique for Diabetic Patients}
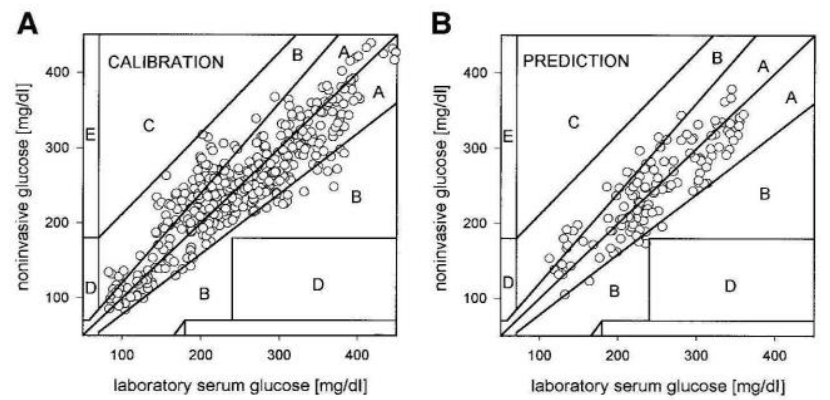

Fig 4. Correlation plot between invasive and noninvasive glucose concentrations for calibration (A) and prediction (B).

\section{VI.RESULT}

The Circuit connection is made between the NIR Transmitter and Receiver. $T$ hen the external voltage of about $5 \mathrm{v}$ is given from the Arduino board. When the finger is placed in between the NIR transmitter and receiver the NIR passes through the finger and then the glucose values were quantified. The final output was exhibited on the serial monitor exhibit.

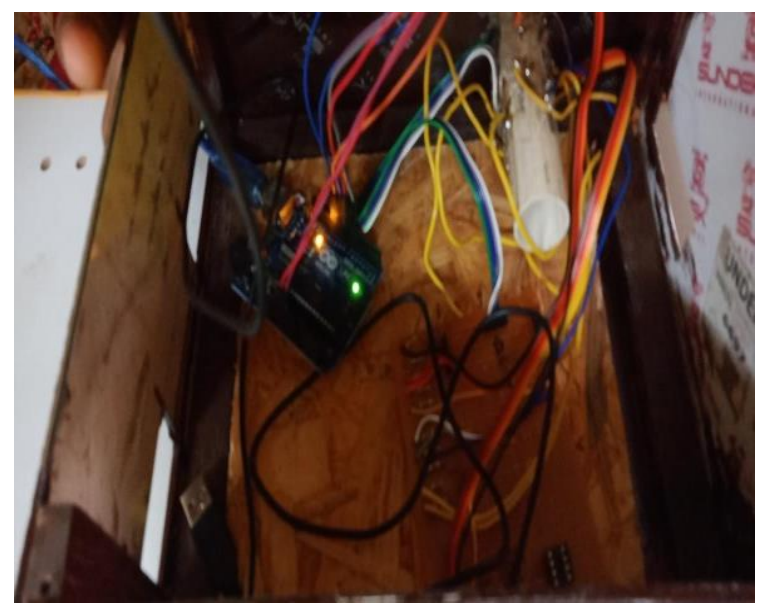

Fig 5. The internal circuit connection of our work

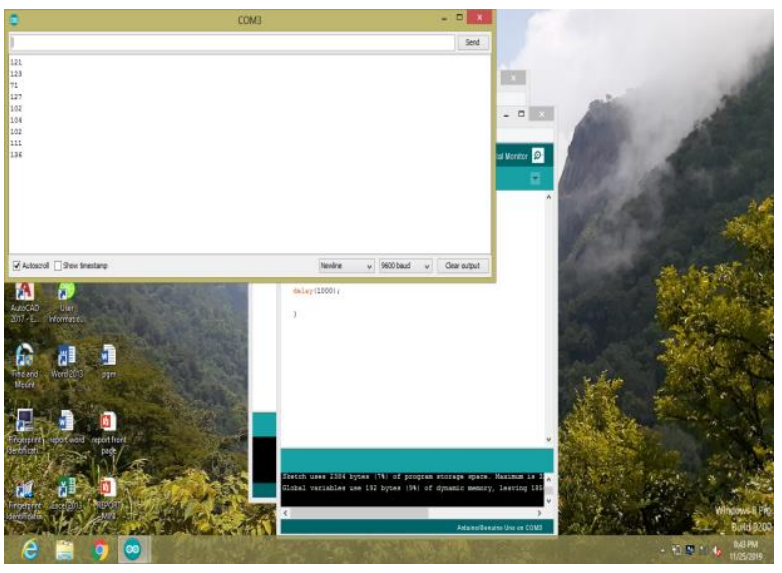

Fig 6 The serial monitor displays the glucose level

\section{CONCLUSION}

In this work, we had tried for a non invasive method for measuring by using the principle known as Beer- Lambert's Law The law states that the amount of light occupied by a substance diffused in a plenarily contagion solvent is equivalent to the fascination of the materialization and the

path length of the light through the clarification.To determine the glucose concentration in the human blood. Here the NIR transceiver is utilized to sense the magnitude of light perforated and some of the light will absorbed predicated on the glucose concentration in the human blood. By processing the absorbed value the output will be exhibited utilizing LCD exhibit.

\section{REFERENCES}

1. Singh, A. K., \& Jha, S. K. (2019). Fabrication and Validation of a Handheld Non-Invasive, Optical Biosensor for Self-Monitoring of Glucose Using Saliva. IEEE Sensors Journal, 19(18), 8332-8339.

2. Volkan Turgul, Izzet Kale "'Simulating the effect of skin thickness and dactylograms to highlights quandary with non-invasive RF blood glucose sensing with fingertip" IEEE Sensor Journal Volume 17, Issue 22.

3. Detection and Relegation of Diabetes Utilizing Modified Convolution Neural Network" IEEE journal of biomedical and health informatics Volume 22,Issue 5.

4. Parag Narkhede, Suraj Dhalwar, B.Karthikeyan "NIR Predicated Non-Invasive Blood Glucose Quantification " ,Indian Journal of Science and Technology Volume 9, Issue 41 ,November 2016.

5. Masab Ahmad, Awis Kamboh \& Ahmed Khan "Non-Invasive Blood Glucose Monitoring Utilizing Near-Infrared Spectroscopy ",EDN Network, October 16,2013.

6. Tura, A., Maran, A., \& Pacini, G. (2007). Non-invasive glucose monitoring: assessment of technologies and devices according to quantitative criteria. Diabetes research and clinical practice, 77(1), 16-40.

7. Singh, A. K., \& Jha, S. K. (2019). Fabrication and Validation of a Handheld Non-Invasive, Optical Biosensor for Self-Monitoring of Glucose Using Saliva. IEEE Sensors Journal, 19(18), 8332-8339.

8. Turgul, V., \& Kale, I. (2017). Simulating the effects of skin thickness and fingerprints to highlight problems with non-invasive RF blood glucose sensing from fingertips. IEEE sensors journal, 17(22), 7553-7560.

9. Lekha, S., \& Suchetha, M. (2017). Real-time non-invasive detection and classification of diabetes using modified convolution neural network. IEEE journal of biomedical and health informatics, 22(5), 1630-1636.

10. R.A. Buda; M. Mohd. Addi “A Portable Non-Invasive Blood Glucometer Device” IEEE Conference On Biomedical Engineering And Sciences, 26 February 2015.

11. Menon, K. U., Hemachandran, D., \& Abhishek, T. K. (2013, April). A survey on non-invasive blood glucose monitoring using NIR. In 2013 International Conference on Communication and Signal Processing (pp. 1069-1072). IEEE.

\section{AUTHORS PROFILE}

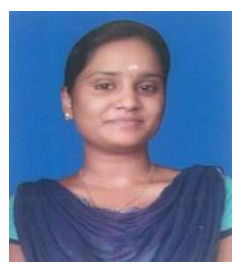

S.Priya Dharshini, is an undergraduate student in department of Electronics and communication Engineering at National Engineering College, Tamil Nadu, India. Her research interest includes IOT, Embedded networking

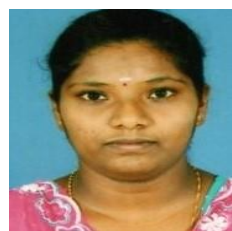

M.Revathy, is an undergraduate student in department of Electronics and communication engineering at National Engineering College, Tamil Nadu, India. Her research interest includes IOT, Embedded networking 


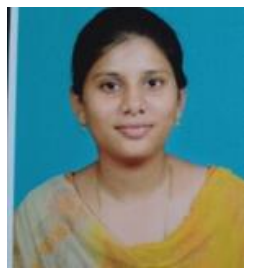

A.Soundharya, an undergraduate student in department of Electronics and communication engineering at National Engineering College, Tamil Nadu, India. Her research interest includes IOT, Embedded networking

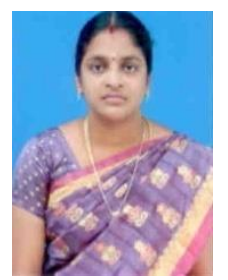

R.Manjula Devi, is an Assistant Professor (SG) in the Department of Electronics and Communication Engineering at National Engineering College, Tamil Nadu, India. he received her B.E. Electronics and Communication Engineering degree from PSR Engineering College, Tamil Nadu in the year 2008. She completed her M.E. Communication systems from Mepco Schlenk Engineering College, Tamil Nadu in 2010. She is currently doing Ph.D. in Anna University, Chennai. She has 9 years of teaching and 3 years of research experience. She has presented two papers in the international conference and received the best paper award. Her research interest is Medical image Processing. She is a life member of IETE \& ISTE society bodies. 